\title{
On memo-viability of fractional equations with the Caputo derivative
}

\author{
Ewa Girejko, Dorota Mozyrska and Małgorzata Wyrwas*
}

${ }^{\text {*Correspondence: }}$ m.wyrwas@pb.edu.pl

Department of Mathematics, Faculty of Computer Science, Bialystok University of Technology, Wiejska 45A, Białystok, 15-351, Poland

\begin{abstract}
In this paper viability results for nonlinear fractional differential equations with the Caputo derivative are proved. We give a necessary condition for fractional viability of a locally closed set with respect to a nonlinear function. A specific sufficient condition is also provided.
\end{abstract}

Keywords: viability; fractional derivative; fractional differential equation

\section{Introduction}

Fractional calculus deals with fractional derivatives and integrals of any order, and it is a field of mathematics that grows out of the traditional definitions of calculus integral and derivative operators. Hence, fractional differential equations are generalizations of ordinary differential equations to equations with an arbitrary order.

In recent years, fractional differential equations have been investigated by many authors [1-6]. However, the problem of viability of fractional differential equations, which consists in finding at least one solution to the equation starting and staying in a constrained set, has not been well developed so far. The classical viability theory has its origin in the Nagumo theorem [7] and is widely exploited starting from ordinary differential equations and reaching differential inclusions based on set-valued maps with a wide range of applications [8-10]. In this paper we continue the subject of the Nagumo theorem for a fractional differential equation with the Caputo derivative. In [11] we showed a sufficient condition for solutions to be viable with respect to a constrained set. In the present paper a necessary condition of viability of a fractional differential equation with the Caputo derivative is proved. It is not trivial to show a necessary condition of viability of a fractional differential equation with the Caputo derivative. Thus we implement the idea that we used in [12] for a fractional differential equation with the Riemann-Liouville derivative, i.e., the initialization problem that leads to a modification of the problem, namely we consider viability of solutions in the memory domain. Then we employ the formula that joins these two types of fractional derivatives. This idea allows us to prove the necessity of the Nagumo theorem going through the classical tools as Bouligand cone, contingent vectors, etc.

The paper is organized as follows. In Section 2 we gather preliminary definitions, notations and some results. Section 3 includes the initialization problem. We formulate the inner value problem with the Caputo derivative on the basis of a similar problem with the Riemann-Liouville derivative. The key result is given in Proposition 7. The last section concerns the viability problem. Theorem 11 and corollaries give necessary conditions of 
viability of a fractional differential equation with the Caputo derivative. Then an illustrative example is provided. Finally Theorem 17 gives a base to formulate a sufficient condition of viability for an equation involving the Caputo derivative that is, however, slightly different from the one that we formulated in [11]. We finish the paper with a block scheme that shows relations among viability conditions presented in the paper.

\section{Preliminaries}

In this section we make a review of notations, definitions, and some preliminary facts, which are useful for the paper. We recall definitions of fractional integrals of arbitrary order, the Caputo and Riemann-Liouville derivatives of order $q \in(0,1)$, and a description of special functions in the fractional calculus.

Definition $1([5,13,14])$ Let $\varphi \in L_{1}\left(\left[0, t_{1}\right], \mathbb{R}\right)$. The integral

$$
\left(I_{0+}^{q} \varphi\right)(t)=\frac{1}{\Gamma(q)} \int_{0}^{t} \varphi(s)(t-s)^{q-1} d s, \quad 0<t \leq t_{1}
$$

where $\Gamma$ is the gamma function and $q>0$, is called the left-sided fractional RiemannLiouville integral of order $q$. Additionally we define $I_{0_{+}}^{0}:=I$ (identity operator).

Remark 2 ([15]) Note that $I_{0+}^{q} f(t)=\left(f * \varphi_{q}\right)(t)$, where $\varphi_{q}(t)=\frac{t^{q-1}}{\Gamma(q)}$ for $t>0, \varphi_{q}(t)=0$ for $t \leq 0$, and $\varphi_{q} \rightarrow \delta(t)$ as $q \rightarrow 0$, with $\delta$ the delta Dirac pseudo function.

Moreover, fractional integration has the following property:

$$
I_{0+}^{q}\left(I_{0+}^{p} \varphi\right)=I_{0+}^{q+p} \varphi, \quad q \geq 0, p \geq 0 .
$$

The best known fractional derivatives are the Riemann-Liouville and the Caputo ones.

Definition $3([5,13])$ Let $\varphi$ be defined on the interval $\left[0, t_{1}\right]$ and $n$ be the natural number satisfying $n=\lfloor q\rfloor+1$ with $\lfloor q\rfloor$ denoting the integer part of $q$. The left-sided RiemannLiouville derivative of order $q$ and the lower limit 0 is defined through the following:

$$
\left(D_{0+}^{q} \varphi\right)(t)=\frac{1}{\Gamma(n-q)}\left(\frac{d}{d t}\right)^{n} \int_{0}^{t} \varphi(s)(t-s)^{n-q-1} d s .
$$

The left-sided Caputo derivative of order $q$ and the lower limit 0 is defined through the following:

$$
\left({ }^{C} D_{0+}^{q} \varphi\right)(t)=\frac{1}{\Gamma(n-q)} \int_{0}^{t} \varphi^{(n)}(s)(t-s)^{n-q-1} d s .
$$

Remark 4 If $q \in(0,1)$, then the left-sided Riemann-Liouville fractional derivative of order $q$ takes the form

$$
\left(D_{0+}^{q} \varphi\right)(t)=\frac{1}{\Gamma(1-q)} \frac{d}{d t} \int_{0}^{t} \varphi(s)(t-s)^{-q} d s=\frac{d}{d t}\left(\left(I_{0+}^{1-q} \varphi\right)(t)\right)
$$


and the left-sided Caputo fractional derivative of order $q$ takes the form

$$
\left({ }^{C} D_{0+}^{q} \varphi\right)(t)=\frac{1}{\Gamma(1-q)} \int_{0}^{t} \varphi^{\prime}(s)(t-s)^{-q} d s=\left(I_{0+}^{1-q} \frac{d}{d s}(\varphi(s))\right)(t) .
$$

If $q \in(0,1]$, then the following comparison formula of the Caputo and RiemannLiouville derivatives holds.

$$
\left({ }^{C} D_{0+}^{q} \varphi\right)(t)=\left(D_{0+}^{q} \varphi\right)(t)-\frac{t^{-q}}{\Gamma(1-q)} \varphi\left(0^{+}\right)
$$

where $\varphi\left(0^{+}\right)=\lim _{t \rightarrow 0^{+}} \varphi(t)$.

From [13], Lemmas 2.4 and 2.5, we have the following properties.

Proposition 5 If $q>0$, then $D_{0+}^{q}\left(I_{0+}^{q} \varphi\right)(t)=\varphi(t)$ for any $\varphi \in L_{1}\left(0, t_{1}\right)$, while

$$
\left(I_{0+}^{q} D_{0+}^{q} \varphi\right)(t)=\varphi(t)
$$

is satisfied for $\varphi \in I_{0+}^{q}\left(L_{1}\left(0, t_{1}\right)\right)$ with

$$
I_{0+}^{q}\left(L_{1}\left(0, t_{1}\right)\right)=\left\{\varphi(t): \varphi(t)=\left(I_{0+}^{q} \psi\right)(t), \psi \in L_{1}\left(0, t_{1}\right)\right\} .
$$

For $q \in(0,1]$ we have

$$
\left(I_{0+}^{q} D_{0+}^{q} \varphi\right)(t)=\varphi(t)-\left.\frac{t^{q-1}}{\Gamma(q)}\left(I_{0+}^{1-q} \varphi\right)(t)\right|_{t=0} .
$$

The following formulas are useful:

$$
I_{0+}^{q} t^{p}=\frac{\Gamma(p+1)}{\Gamma(p+q+1)} t^{p+q} \quad \text { and } \quad D_{0+}^{q} t^{p}=\frac{\Gamma(p+1)}{\Gamma(p-q+1)} t^{p-q},
$$

in particular,

$$
I_{0+}^{q} 1=\frac{t^{q}}{\Gamma(q+1)}, \quad D_{0+}^{q} 1=\frac{t^{-q}}{\Gamma(1-q)}, \quad{ }^{C} D_{0+}^{q} 1=0 .
$$

\section{The inner value problem}

Let us consider the fractional differential equations with the Caputo fractional derivative

$$
\left({ }^{C} D_{0+}^{q} x\right)(t)=f(t, x(t)), \quad 0<q<1, t \in(0, T],
$$

with $x:(0, T] \rightarrow \mathbb{R}^{n}$, satisfying the inner condition

$$
x\left(t_{0}\right)=x_{0} \in \mathbb{R}^{n},
$$

where $t_{0} \in(0, T)$. By (2) equation (3) can be rewritten with the Riemann-Liouville fractional derivative as follows:

$$
\left(D_{0+}^{q} x\right)(t)=f(t, x(t))+\frac{t^{-q}}{\Gamma(1-q)} x\left(0^{+}\right),
$$

where for $0<q<1, t \in(0, T]$. 
Let us define the function $g:(0, T] \times \mathbb{R}^{n} \rightarrow \mathbb{R}^{n}$ as $g(t, x(t)):=f(t, x(t))+\frac{t^{-q}}{\Gamma(1-q)} x\left(0^{+}\right)$. Then we get

$$
\left(D_{0+}^{q} x\right)(t)=g(t, x(t)), \quad t \in(0, T] .
$$

Assume that (6) satisfies the boundary inner condition

$$
x\left(t_{0}\right)=x_{0} \in \mathbb{R}^{n}, \quad 0<t_{0}<T .
$$

From [13] we know the form of the Volterra fractional integral for $q \in(0,1)$

$$
x(t)=\left(I_{0+}^{q} g(s, x(s))\right)(t)+\left(\frac{t_{0}}{t}\right)^{1-q} \cdot\left(x_{0}-\left(I_{0+}^{q} g(s, x(s))\right)\left(t_{0}\right)\right) .
$$

Then

$$
\begin{aligned}
x(t)= & \left(I_{0+}^{q} f(s, x(s))\right)(t)+\left(\frac{t_{0}}{t}\right)^{1-q} \cdot\left(x_{0}-\left(I_{0+}^{q} f(s, x(s))\right)\left(t_{0}\right)\right) \\
& +x\left(0^{+}\right)\left(1-\left(\frac{t_{0}}{t}\right)^{1-q}\right) .
\end{aligned}
$$

Remark 6 Since for $x\left(0^{+}\right)=0$ we have $f \equiv g$, we look for the solutions of (6) in the set of functions $x(\cdot)$ such that $x\left(0^{+}\right) \neq 0$.

Using the fractional integral $I_{0+}^{1-q}$ for both sides of (9) and applying formula (1), we get

$$
\begin{aligned}
\left(I_{0+}^{1-q} x\right)(t)= & \left(I_{0+}^{1} f(s, x(s))\right)(t) \\
& +\left(I_{0+}^{1-q}\left(\frac{t_{0}}{s}\right)^{1-q}\right)(t) \cdot\left(x_{0}-\left(I_{0+}^{q} f(s, x(s))\right)\left(t_{0}\right)\right) \\
& +x\left(0^{+}\right)\left(I_{0+}^{1-q}\left(1-\left(\frac{t_{0}}{s}\right)^{1-q}\right)\right)(t) .
\end{aligned}
$$

Moreover, as $\left(I_{0+}^{1-q}\left(\frac{t_{0}}{s}\right)^{1-q}\right)(t)=t_{0}^{1-q} \frac{\Gamma(q)}{\Gamma(1-q+q)} q^{q+1-q-1}=\Gamma(q) t_{0}^{1-q}$, then from (10) we have that

$$
\begin{aligned}
\left(I_{0+}^{1-q} x\right)(t)= & \int_{0}^{t} f(s, x(s)) d s+\Gamma(q) t_{0}^{1-q}\left(x_{0}-\left(I_{0+}^{q} f(s, x(s))\right)\left(t_{0}\right)\right) \\
& +x\left(0^{+}\right)\left(\frac{t^{1-q}}{\Gamma(2-q)}-\Gamma(q) t_{0}^{1-q}\right) .
\end{aligned}
$$

Let us put $m\left(t_{0}, t\right):=\left(I_{0+}^{1-q} x\right)(t)$, then $\frac{d}{d t} m\left(t_{0}, t\right)=g(t, x(t)),\left.\frac{d}{d t} m\left(t_{0}, t\right)\right|_{t=t_{0}}=\left(D_{0+}^{q} x\right)\left(t_{0}\right)=$ $g\left(t_{0}, x\left(t_{0}\right)\right)$.

In formulas (9)-(11) we have the value $x\left(0^{+}\right)$as an additional parameter. As we are going to consider the initialization process and its viability in the memory domain, it is important to consider assumptions connected with the memory function $m\left(t_{0}, \cdot\right)$. We claim that $\lim _{t \rightarrow 0^{+}} m\left(t_{0}, t\right)=0$. Further, we observe that taking the limit of (11) with $t$ tending to zero one gets the following:

$$
\left(I_{0^{+}}^{q} f(s, x(s))\right)\left(t_{0}\right)=x\left(t_{0}\right)-x\left(0^{+}\right) .
$$


Calculating values of $f(s, x(s))$ for $s \in\left(0, t_{0}\right]$ one finds some difficulties in predicting values of the memory in the light of viability, thus we consider the modification of the problem that leads to the main results.

Let us consider a new inner value problem

$$
D_{0+}^{q} x(t)=\widetilde{g}(t, x(t)), \quad 0<q<1, t \in(0, T]
$$

satisfying the inner condition

$$
x\left(t_{0}\right)=x_{0} \in \mathbb{R}^{n}, \quad 0<t_{0}<T,
$$

where

$$
\tilde{g}(t, x(t))= \begin{cases}g\left(t_{0}, x_{0}\right), & t \in\left[0, t_{0}\right), \\ g(t, x(t)), & t \geq t_{0} .\end{cases}
$$

Function $\widetilde{g}$ is still continuous if $g$ is continuous. Let us denote by $\widetilde{x}(\cdot)$ the solution of the inner value problem (13)-(14), and let $\widetilde{m}\left(t_{0}, t\right):=\left(I_{0+}^{1-q} \widetilde{x}\right)(t)$. For $t \in\left(0, t_{0}\right]$ we have the formula of the solution:

$$
\widetilde{x}(t)=\frac{t^{q}-t_{0} \cdot t^{q-1}}{\Gamma(q+1)}\left(f\left(t_{0}, x_{0}\right)+\frac{t_{0}^{-q}}{\Gamma(1-q)} x\left(0^{+}\right)\right)+\left(\frac{t_{0}}{t}\right)^{1-q} \cdot x_{0}
$$

and

$$
\widetilde{m}\left(t_{0}, t\right)=\left(t-\frac{t_{0}}{q}\right) \cdot\left(f\left(t_{0}, x_{0}\right)+\frac{t_{0}^{-q}}{\Gamma(1-q)} x\left(0^{+}\right)\right)+\Gamma(q) t_{0}^{1-q} x_{0} .
$$

Then $\left(D_{0+}^{q} \widetilde{x}\right)(t)=g\left(t_{0}, x_{0}\right), \widetilde{x}\left(t_{0}\right)=x_{0}$ and

$$
\widetilde{m}\left(t_{0}, t_{0}\right)=\frac{q-1}{q} t_{0} f\left(t_{0}, x_{0}\right)+\Gamma(q) t_{0}^{1-q} x_{0}+\frac{q-1}{q \Gamma(1-q)} t_{0}^{1-q} x\left(0^{+}\right) .
$$

Observe that $\lim _{t_{0} \rightarrow 0^{+}} \tilde{m}\left(t_{0}, t_{0}\right)=0, \lim _{q \rightarrow 1} \tilde{m}\left(t_{0}, t_{0}\right)=x_{0}$. The steps presented for the new function $\widetilde{g}$ are useful if we need to know the value of $m_{0}:=m\left(t_{0}, t_{0}\right)=\left(I_{0+}^{1-q} x\right)\left(t_{0}\right)$.

Proposition 7 Let $f$ be bounded on $[0, T]$. For any $\varepsilon>0$, there is $0<t_{0}<T$ such that

$$
\left\|\widetilde{m}\left(t_{0}, t\right)-m\left(t_{0}, t\right)\right\| \leq \varepsilon, \quad t \in\left[t_{0}, T\right] .
$$

Proof Let us take $\varepsilon>0$ and $\|f(t, x(t))\| \leq M$ for $t \in[0, T]$. Then for $0<t_{0} \leq t<T$ we have $\|g(t, x(t))\|=\left\|f(t, x(t))+\frac{t^{-q}}{\Gamma(1-q)} x\left(0^{+}\right)\right\| \leq\|f(t, x(t))\|+\frac{t^{-q}}{\Gamma(1-q)}\left\|x\left(0^{+}\right)\right\| \leq M+\frac{t_{0}^{-q}}{\Gamma(1-q)}\left\|x\left(0^{+}\right)\right\|$and

$$
\begin{aligned}
\left\|\tilde{m}\left(t_{0}, t\right)-m\left(t_{0}, t\right)\right\|= & \| \int_{0}^{t_{0}}\left(g\left(t_{0}, x_{0}\right)-g(s, x(s))\right) d s \\
& -\Gamma(q) t_{0}^{1-q}\left(I_{0+}^{q} g\left(t_{0}, x_{0}\right)\right)\left(t_{0}\right)+\Gamma(q) t_{0}^{1-q}\left(I_{0+}^{q} g(s, x(s))\right)\left(t_{0}\right) \| \\
\leq & \left\|t_{0} g\left(t_{0}, x_{0}\right)-\Gamma(q) t_{0}^{1-q}\left(I_{0+}^{q} g\left(t_{0}, x_{0}\right)\right)\left(t_{0}\right)\right\|
\end{aligned}
$$




$$
\begin{aligned}
& +\left\|\Gamma(q) t_{0}^{1-q}\left(I_{0+}^{q} g(s, x(s))\right)\left(t_{0}\right)-\int_{0}^{t_{0}} g(s, x(s)) d s\right\| \\
= & t_{0} \frac{1-q}{q}\left\|g\left(t_{0}, x_{0}\right)\right\| \\
& +\left\|\Gamma(q) t_{0}^{1-q}\left(I_{0+}^{q} g(s, x(s))\right)\left(t_{0}\right)-\int_{0}^{t_{0}} g(s, x(s)) d s\right\| \\
\leq & t_{0} \frac{1-q}{q}\left(M+\frac{t_{0}^{-q}}{\Gamma(1-q)}\left\|x\left(0^{+}\right)\right\|\right) \\
& +\left(M+\frac{t_{0}^{-q}}{\Gamma(1-q)}\left\|x\left(0^{+}\right)\right\|\right) \cdot\left|\Gamma(q) t_{0}^{1-q} \frac{t_{0}^{q}}{\Gamma(1+q)}-t_{0}\right| \\
= & 2 t_{0} \frac{1-q}{q}\left(M+\frac{t_{0}^{-q}}{\Gamma(1-q)}\left\|x\left(0^{+}\right)\right\|\right) .
\end{aligned}
$$

Then for

$$
t_{0} \leq \min \left(\frac{q \varepsilon}{4(1-q) M},\left[\frac{q \Gamma(1-q) \varepsilon}{4(1-q)\left\|x\left(0^{+}\right)\right\|}\right]^{\frac{1}{1-q}}\right)
$$

we get

$$
\left\|\widetilde{m}\left(t_{0}, t\right)-m\left(t_{0}, t\right)\right\| \leq \frac{2(1-q) M}{q} t_{0}+\frac{2(1-q)\left\|x\left(0^{+}\right)\right\|}{q \Gamma(1-q)} t_{0}^{1-q} \leq \varepsilon .
$$

\section{Viability problem}

Before going to viability terms, we set some notations. For $\varepsilon>0$, by $\varepsilon$-neighborhood of a set $K \subset \mathbb{R}^{n}$ we mean the following:

$$
K^{\varepsilon}:=\left\{x \in \mathbb{R}^{n}: \operatorname{dist}(x, K)<\varepsilon\right\} .
$$

Let us define the distance between two sets $A \subset \mathbb{R}^{n}$ and $B \subset \mathbb{R}^{n}$ as $\triangle(A, B):=\sup \{\operatorname{dist}(q, B)$ : $q \in A\}$. Note that $\triangle(A, B)$ is not the usual symmetric distance between two sets. Indeed, if $A \subset B$ then $\triangle(A, B)=0$ while $\triangle(B, A) \neq 0$.

By definitions of $m, \widetilde{m}$ and Proposition 7 the following proposition is obvious.

Proposition 8 Let $f$ be bounded on $[0, T]$. Let us take $\varepsilon>0$ and $t_{0} \in(0, T]$ such that

$$
\left\|\tilde{m}\left(t_{0}, t\right)-m\left(t_{0}, t\right)\right\|<\varepsilon
$$

Then

$$
\triangle\left(\operatorname{Graph}\left(m\left(t_{0}, \cdot\right)\right),\left[t_{0}, T\right] \times K\right)=0 \Leftrightarrow \Delta\left(\operatorname{Graph}\left(\tilde{m}\left(t_{0}, \cdot\right)\right),\left[t_{0}, T\right] \times K\right)<\varepsilon
$$

which we can write equivalently

$$
m\left(t_{0}, t\right) \in K \quad \Leftrightarrow \quad \tilde{m}\left(t_{0}, t\right) \in K^{\varepsilon} \quad \text { for all } t \in\left[t_{0}, T\right] .
$$

Similarly as for the ordinary differential equations (see [16]), one can define the viability of a subset with respect to the fractional differential equation (3).

Let us denote by $I$ an open interval in $\mathbb{R}$. 
Definition 9 Let $K \subset \mathbb{R}^{n}$ be a nonempty locally closed set and $f: I \times K \rightarrow \mathbb{R}^{n}$. The subset $K$ is fractionally memo-viable with respect to $f$ if for any $\left(t_{0}, x_{0}\right) \in I \times K$ equation (3) has at least one solution $x:\left[t_{0}, T\right] \rightarrow \mathbb{R}^{n}$ satisfying $m\left(t_{0}, t\right) \in K$ for $t \in\left[t_{0}, T\right]$, where $t_{0}>0$.

The idea of viability of fractional differential equations can be expressed using the concept of tangent cone. There are many notions of tangency of a vector to a set (see, for example, [16], Section 2.3). We will follow the concept of contingent vectors (see [9]).

Let us recall that for $K \subset \mathbb{R}^{n}$ and $x_{0} \in K$ one can define the vector tangent to the set $K$ as follows.

Definition 10 The vector $\eta \in \mathbb{R}^{n}$ is contingent to the set $K$ at the point $x_{0}$ if

$$
\liminf _{h \downarrow 0} \frac{1}{h} \operatorname{dist}\left(x_{0}+h \eta ; K\right)=0 .
$$

The set of all vectors that are contingent to the set $K$ at point $x_{0}$ is a closed cone, see [16], Proposition 2.3.1. This cone, denoted by $\mathcal{T}_{K}\left(x_{0}\right)$, is called contingent cone (Bouligand cone) to the set $K$ at $x_{0} \in K$. From [16], Proposition 2.3.2, we know that $\eta \in \mathcal{T}_{K}\left(x_{0}\right)$ if and only if for every $\varepsilon>0$ there exist $h \in(0, \varepsilon)$ and $p_{h} \in B(0, \varepsilon)$ such that $x_{0}+h\left(\eta+p_{h}\right) \in K$, where $B(0, \varepsilon)$ denotes the closed ball in $\mathbb{R}^{n}$ centered at 0 and of radius $\varepsilon>0$.

Theorem 11 Let $K \subset \mathbb{R}^{n}$ be nonempty and $f: I \times K \rightarrow \mathbb{R}^{n}$. If the subset $K$ is fractionally memo-viable with respect to $f$, then $g\left(t_{0}, x_{0}\right) \in \mathcal{T}_{K}\left(m_{0}\right)$, where $x_{0}=x\left(t_{0}\right)$ and $m_{0}=$ $m\left(t_{0}, t_{0}\right)=\left(I_{0+}^{1-q} x\right)\left(t_{0}\right)$.

Proof Let $\left(t_{0}, m_{0}\right) \in I \times K$ and $K$ be fractionally viable of order $q$ with respect to $f$. Then there is $T \in I, T>t_{0}$, and a function $x:\left[t_{0}, T\right] \rightarrow K$ satisfying $\left(I_{0+}^{1-q} x\right)\left(t_{0}\right)=m_{0}$ and $D_{0+}^{q} x(t)=f(t, x(t))$ for every $t \in\left[t_{0}, T\right]$. Moreover we have

$$
\begin{aligned}
& \lim _{h \downarrow 0} \frac{1}{h}\left\|\left(I_{0+}^{1-q} x\right)\left(t_{0}\right)+h g\left(t_{0}, x_{0}\right)-I_{0+}^{1-q} x\left(t_{0}+h\right)\right\| \\
& \quad=\lim _{h \downarrow 0}\left\|g\left(t_{0}, x_{0}\right)-\frac{\left(I_{0+}^{1-q} x\right)\left(t_{0}+h\right)-\left(I_{0+}^{1-q} x\right)\left(t_{0}\right)}{h}\right\| \\
& \quad=\left\|g\left(t_{0}, x_{0}\right)-D_{0+}^{q} x\left(t_{0}\right)\right\|=0 .
\end{aligned}
$$

The above calculation shows that, for every $\left(t_{0}, m_{0}\right) \in I \times K, g\left(t_{0}, x_{0}\right) \in \mathcal{T}_{K}\left(m_{0}\right)$ and the proof is complete.

Note that it is difficult to check the condition $g\left(t_{0}, x_{0}\right) \in \mathcal{T}_{K}\left(m_{0}\right)$ in Theorem 11. The above theorem is still true for the initial inner value problem with the right-hand side of $\widetilde{g}$ given by (15). Let

$$
\tilde{f}(t, x(t))= \begin{cases}f\left(t_{0}, x_{0}\right), & t \in\left[0, t_{0}\right) \\ f(t, x(t)), & t \geq t_{0}\end{cases}
$$

Then the following is true. 
Corollary 12 Let $K \subset K^{\varepsilon} \subset \mathbb{R}^{n}$ and $f: I \times K \rightarrow \mathbb{R}^{n}$. If the subset $K$ is fractionally memoviable with respect to $f$, then $g\left(t_{0}, x_{0}\right) \in \mathcal{T}_{K^{\varepsilon}}\left(\widetilde{m}_{0}\right)$.

From Theorem 11 we get the following weaker result.

Corollary 13 Let $K \subset \mathbb{R}^{n}$ be nonempty and $f: I \times K \rightarrow \mathbb{R}^{n}$. If the subset $K$ is fractionally memo-viable with respect to $f$, then $f\left(t_{0}, x_{0}\right) \in \mathcal{T}_{K}\left(m_{0}\right)$ or $x\left(0^{+}\right) \in \mathcal{T}_{K}\left(m_{0}\right)$, where $x_{0}=x\left(t_{0}\right)$ and $m_{0}=m\left(t_{0}, t_{0}\right)=\left(I_{0+}^{1-q} x\right)\left(t_{0}\right)$.

Example 14 Let us consider a one-dimensional problem with the set $K=\mathbb{R}_{+} \cup\{0\}$ and $f(t, x(t))=c=$ const. Then $g(t, x(t))=c+\frac{t^{-q}}{\Gamma(1-q)} x\left(0^{+}\right)$for $0<t<T$ and

$$
\tilde{g}(t, x(t))= \begin{cases}c+\frac{t_{0}^{-q}}{\Gamma(1-q)} x\left(0^{+}\right) & \text {for } 0<t<t_{0} \\ c+\frac{t^{-q}}{\Gamma(1-q)} x\left(0^{+}\right) & \text {for } t_{0} \leq t<T\end{cases}
$$

Moreover, for $x_{0}=x\left(t_{0}\right)$, we have

$$
x(t)=\frac{c}{\Gamma(1+q)} t^{q}\left(1-\frac{t_{0}}{t}\right)+x_{0}\left(\frac{t_{0}}{t}\right)^{1-q}+x\left(0^{+}\right)\left[1-\left(\frac{t_{0}}{t}\right)^{1-q}\right]
$$

and $m_{0}=m\left(t_{0}, t_{0}\right)=c t_{0}\left(1-\frac{1}{q}\right)+t_{0}^{1-q} \Gamma(q)\left[x_{0}+x\left(0^{+}\right)\left(\frac{1}{\Gamma(q) \Gamma(2-q)}-1\right)\right]$. Let us take $m_{0} \geq 0$, then

$$
\mathcal{T}_{K}\left(m_{0}\right)= \begin{cases}\mathbb{R} & \text { for } m_{0}>0 \\ {[0,+\infty)} & \text { for } m_{0}=0\end{cases}
$$

We will show that if $g\left(t_{0}, x_{0}\right) \notin \mathcal{T}_{K}\left(m_{0}\right)$, then $K$ is not memo-viable. Since for $m_{0}>0$ we have $\mathcal{T}_{K}\left(m_{0}\right)=\mathbb{R}$, it is nothing to show then.

Let $m_{0}=0$, then $g\left(t_{0}, x_{0}\right)<0$, i.e., $c+\frac{t_{0}^{-q}}{\Gamma(1-q)} x\left(0^{+}\right)<0$. Let us notice that the term $\frac{t_{0}^{-q}}{\Gamma(1-q)}$ is positive.

Let $x\left(0^{+}\right)=0$, then obviously $c<0$, and we get

$$
x(t)=c \frac{t^{q}}{\Gamma(1+q)}\left(1-q \frac{t_{0}}{t}\right)<0 \quad \text { if only } t_{0} \leq t
$$

Integrating the last term, on the basis of monotonicity of integral one gets

$$
m\left(t_{0}, t\right)=\left(I_{0^{+}}^{1-q} x\right)(t)=c\left(t-t_{0}\right)<0 .
$$

Now let $x\left(0^{+}\right)>0$ and since $g\left(t_{0}, x_{0}\right)<0$, which means that $c<\frac{-t_{0}^{-q}}{\Gamma(1-q)} x\left(0^{+}\right)$, thus $c<0$. Therefore one can show the following:

$$
m\left(t_{0}, t\right)=c t+x\left(0^{+}\right)\left(1-q \frac{t_{0}}{t}\right) t_{0}^{1-q}<0
$$

provided $t>\frac{x\left(0^{+}\right)}{c}\left(\frac{1}{\Gamma(q) \Gamma(2-q)}-1\right) t_{0}^{1-q}$. 
Finally, let us assume $x\left(0^{+}\right)<0$, and since $g\left(t_{0}, x_{0}\right)<0$, we get $c<\frac{-t_{0}^{-q}}{\Gamma(1-q)} x\left(0^{+}\right)$. Again one can show the following:

$$
m\left(t_{0}, t\right)=c t+x\left(0^{+}\right)\left(1-q \frac{t_{0}}{t}\right) t_{0}^{1-q}<0
$$

if only $t_{0}<t<\left(1-\frac{1}{\Gamma(q) \Gamma(2-q)}\right) \Gamma(1-q) t_{0}$. Since $m\left(t_{0}, t\right)<0$, it follows that $K$ is not memoviable with respect to $f$.

Finally, in order to prove the sufficient condition of viability, we need the following definition and proposition.

Definition 15 ([11]) Let $K \subset \mathbb{R}^{n}$ be nonempty and $f: I \times K \rightarrow \mathbb{R}^{n}$. The subset $K$ is fractionally viable with respect to $f$ if for any $\left(t_{0}, x_{0}\right) \in I \times K$ equation (3) has at least one solution $x:\left[t_{0}, T\right] \rightarrow K$ satisfying $x\left(t_{0}\right)=x_{0}$. Such a solution we call viable with respect tof.

Proposition 16 ([11]) Let $K \subset \mathbb{R}^{n}$ be a nonempty and locally closed set, and let $f: I \times K \rightarrow$ $\mathbb{R}^{n}$ be a vector-valued continuous function. If $f\left(t_{0}, x_{0}\right) \in \mathcal{T}_{K}\left(x_{0}\right)$ for every $\left(t_{0}, x_{0}\right) \in I \times K$, then $K$ is fractionally viable with respect to $f$.

Let us re-scale and shift the set $K^{\varepsilon}$ in such a way that elements of this set are again from the domain of $f$, namely

$$
\tilde{K}^{\varepsilon}=\frac{t_{0}^{q-1}}{\Gamma(q)} K^{\varepsilon}+\frac{1-q}{\Gamma(q+1)} t_{0}^{q} f\left(t_{0}, x_{0}\right)+\frac{1-q}{\Gamma(q+1) \Gamma(1-q)} x\left(0^{+}\right) .
$$

To formulate next theorem, which is a middle step in getting a certain sufficient condition of viability, we use the notion (19).

Theorem 17 Let $K \subset K^{\varepsilon} \subset \mathbb{R}^{n}$ be nonempty and $f: I \times K \rightarrow \mathbb{R}^{n}$. If the subset $K$ is fractionally memo-viable with respect to $f$, then $g\left(t_{0}, x_{0}\right) \in \mathcal{T}_{\tilde{K}^{\varepsilon}}\left(x_{0}\right)$, where $x_{0}=x\left(t_{0}\right)$.

Proof Let $\left(t_{0}, m_{0}\right) \in I \times K$ and $K$ be fractionally memo-viable with respect to $f$. Then Corollary 12 gives $g\left(t_{0}, x_{0}\right) \in \mathcal{T}_{K^{\varepsilon}}\left(\tilde{m}_{0}\right)$. The latter means that

$$
\liminf _{h \downarrow 0} \frac{1}{h} \operatorname{dist}\left(\tilde{m}_{0}+h g\left(t_{0}, x_{0}\right), K^{\varepsilon}\right)=0 .
$$

By formula (16) we can rewrite the above equation in the following way:

$$
\min _{y \in K^{\varepsilon}} \frac{1}{h}\left|\frac{q-1}{q} t_{0} f\left(t_{0}, x_{0}\right)+\Gamma(q) t_{0}^{1-q} x_{0}+\frac{q-1}{q \Gamma(1-q)} t_{0}^{1-q} x\left(0^{+}\right)+h g\left(t_{0}, x_{0}\right)-y\right| \rightarrow 0 .
$$

Let $\tilde{K}^{\varepsilon}$ be as it is given in (19), i.e.,

$$
\tilde{K}^{\varepsilon}=\frac{t_{0}^{q-1}}{\Gamma(q)} K^{\varepsilon}+\frac{1-q}{\Gamma(q+1)} t_{0}^{q} f\left(t_{0}, x_{0}\right)+\frac{1-q}{\Gamma(q+1) \Gamma(1-q)} x\left(0^{+}\right),
$$




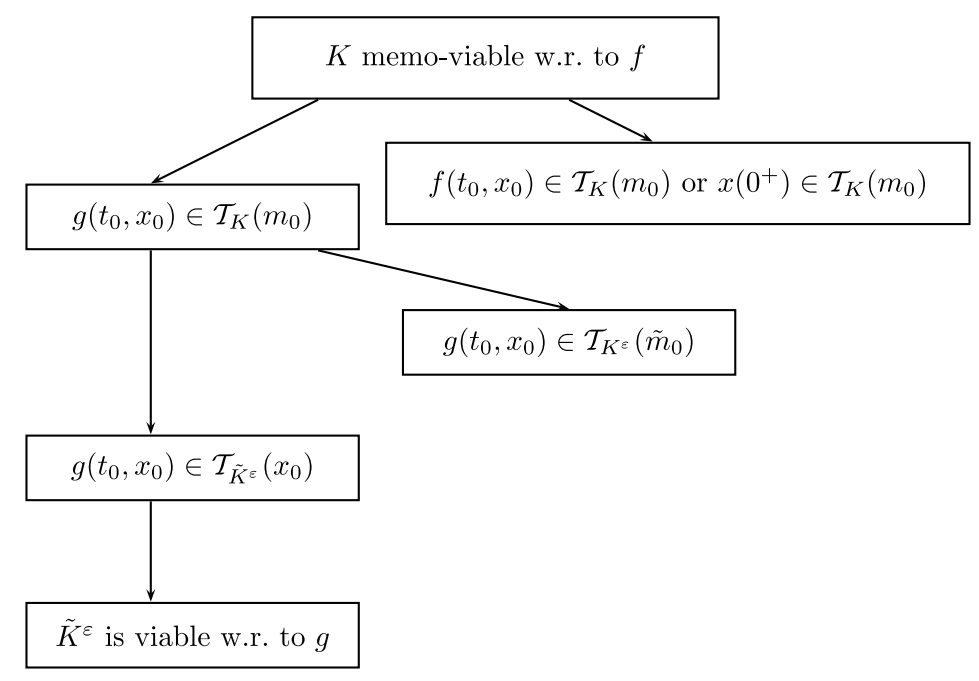

Figure 1 Block scheme concerning the viability conditions.

then for $\tilde{y}=\frac{q-1}{\Gamma(q+1)} t_{0}^{q} f\left(t_{0}, x_{0}\right)+\frac{q-1}{\Gamma(q+1) \Gamma(1-q)} x\left(0^{+}\right)-\frac{t_{0}^{q-1}}{\Gamma(q)} y$ and $\tilde{h}=\frac{\Gamma(q) t_{0}^{1-q}}{h}$, we get $\tilde{y} \in \tilde{K}^{\varepsilon}$ and $\tilde{h} \rightarrow 0$ when $h \rightarrow 0$, thus

$$
\min _{\tilde{y} \tilde{K}^{\varepsilon}} \frac{1}{\tilde{h}}\left|x_{0}+\tilde{h} g\left(t_{0}, x_{0}\right)-y\right| \longrightarrow 0
$$

while $\tilde{h} \rightarrow 0$. Therefore we get $g\left(t_{0}, x_{0}\right) \in \mathcal{T}_{\tilde{K}^{\varepsilon}}\left(x_{0}\right)$.

The next corollary gives, in fact, a sufficient condition of viability for an equation involving the Caputo derivative that is, however, slightly different from the one that we formulated in [11].

Corollary 18 Let $K \subset K^{\varepsilon} \subset \mathbb{R}^{n}$ be nonempty and $f: I \times \mathbb{R}^{n} \rightarrow \mathbb{R}^{n}$. Let $\tilde{K}^{\varepsilon}=\frac{t_{0}^{q-1}}{\Gamma(q)} K^{\varepsilon}+$ $\frac{1-q}{\Gamma(q+1)} t_{0}^{q} f\left(t_{0}, x_{0}\right)+\frac{1-q}{\Gamma(q+1) \Gamma(1-q)} x\left(0^{+}\right)$. If $g\left(t_{0}, x_{0}\right) \in \mathcal{T}_{\tilde{K}^{\varepsilon}}\left(x_{0}\right)$, where $x_{0}=x\left(t_{0}\right)$, then $\tilde{K}^{\varepsilon}$ is viable with respect to $g$.

Proof The thesis is a simple consequence of Proposition 16 and Theorem 17.

\section{Conclusions}

As a conclusion we propose a block scheme (see Figure 1) that shows relations among viability conditions for sets $K, K^{\varepsilon}$ and $\tilde{K}^{\varepsilon}$, where $K \subset K^{\varepsilon} \subset \mathbb{R}^{n}$ and $\tilde{K}^{\varepsilon}=\frac{t_{0}^{q-1}}{\Gamma(q)} K^{\varepsilon}+$ $\frac{1-q}{\Gamma(q+1)} t_{0}^{q} f\left(t_{0}, x_{0}\right)+\frac{1-q}{\Gamma(q+1) \Gamma(1-q)} x\left(0^{+}\right)$for $f$ and $g$ being the right-hand sides of equations (3) and (6), respectively. 


\section{Acknowledgements}

The authors are very grateful to anonymous reviewers for valuable suggestions and comments, which improved the quality of the paper. The work was supported by Bialystok University of Technology grant S/WI/2/2011.

Received: 21 October 2014 Accepted: 3 February 2015 Published online: 24 February 2015

\section{References}

1. Almeida, $R$, Torres, DFM: Necessary and sufficient conditions for the fractional calculus of variations with Caputo derivatives. Commun. Nonlinear Sci. Numer. Simul. 16(3), 1490-1500 (2011)

2. Abdeljawad, T, Baleanu, D: Fractional differences and integration by parts. J. Comput. Anal. Appl. 13(3), 574-582 (2011)

3. Bai, Z, Lü, H: Positive solutions for boundary value problems of nonlinear fractional differential equations. J. Math. Anal. Appl. 311(2), 495-505 (2005)

4. Zhang, S: Existence of a positive solution for some class of nonlinear fractional differential equations. J. Math. Anal. Appl. 278, 136-148 (2003)

5. Podlubny, I: Fractional Differential Equations. Mathematics in Sciences and Engineering, vol. 198. Academic Press, San Diego (1999)

6. Miller, KS, Ross, B: Fractional difference calculus. In: Proceedings of the International Symposium on Univalent Functions, Fractional Calculus and Their Applications, Nihon University, Kōriyama, Japan, pp. $139-152$ (1988)

7. Nagumo, N: Über die lage der integralkurven gewöhnlicher differentialgleichungen. Proc. Phys. Math. Soc. Jpn. 24, 551-559 (1942)

8. Aubin, JP: Viability Theory. Birkhäuser, Berlin (1991)

9. Aubin, JP, Frankowska, H: Set-Valued Analysis. Birkhäuser Boston, Boston (1990)

10. Aubin, JP, Bayen, AM, Saint-Pierre, P: Viability Theory, New Directions. Springer, Berlin (2011)

11. Girejko, E, Mozyrska, D, Wyrwas, M: A sufficient condition of viability for fractional differential equations with the Caputo derivative. J. Math. Anal. Appl. 381, 146-154 (2011)

12. Mozyrska, D, Girejko, E, Wyrwas, M: A necessary condition of viability for fractional differential equations with initialization. Comput. Math. Appl. 62(9), 3642-3647 (2011)

13. Kilbas, AA, Srivastava, HM, Trujillo, JJ: Theory and Applications of Fractional Differential Equations. North-Holland Mathematics Studies, vol. 204. Elsevier, Amsterdam (2006)

14. Samko, SG, Kilbas, AA, Marichev, OI: Fractional Integrals and Derivatives: Theory and Applications. Gordon \& Breach Yverdon (1993)

15. Benchora, M, Hamani, S, Ntouyas, SK: Boundary value problems for differential equations with fractional order. Surv. Math. Appl. 3, 1-12 (2008)

16. Cañada, A, Drábek, P, Fonda, A: Handbook of Differential Equations: Ordinary Differential Equations. Elsevier, Amsterdam (2005)

\section{Submit your manuscript to a SpringerOpen ${ }^{0}$ journal and benefit from:}

- Convenient online submission

- Rigorous peer review

- Immediate publication on acceptance

- Open access: articles freely available online

- High visibility within the field

- Retaining the copyright to your article 